\title{
Local denaturation of collagen fibres during the mechanical rupture of collagenous fibrous tissue*
}

\author{
R. J. MINNS ${ }^{1}$, AND F.S. STEVEN 2 \\ From the ${ }^{1}$ Department of Engineering Science, University of Durham, Durham, and the ${ }^{2}$ Department of Medical \\ Biochemistry, University of Manchester
}

SUMMARY Human extensor tendons of the hand were pulled to rupture, and the torn ends, when observed in the scanning electron microscope, appeared to be knotted, possibly owing to the denaturation of the collagen. This was confirmed by fluorimetry assays of both the ruptured ends and the middle unbroken sections of the same tendons. The use of a proteolytic enzyme, trypsin, to remove the denatured material and enhance the repair of organised collagen fibres from both ends is suggested if the ruptured ends have the denatured knotted appearance commonly observed clinically.

Tendons that have become ruptured in vivo have usually failed because of 2 conditions: because an unusually large force has been applied through the tendon before muscular control has resisted the load, or because the tendon, in particular the collagen fibre network, which resists tensile loads transmitted through the tendon, has undergone biochemical attack substantially weakening the collagen. Tendons that have become divided as a result of trauma show a lesion at the rupture in which the ends have become atrophic and rounded producing a blunt amputation stump. After several months of repair histological evidence shows the junction of the new and old tendon and no regeneration from the proximal stump (Peacock, 1977).

Clinical evidence and our interests in degeneration of collagenous tissue led to us conduct a preliminary study on the morphology of tendon ends after mechanical rupture (Steven et al., 1975). A hypothesis we put forward during that study was that the appearance of the ruptured end was a result of denaturation at the fibre ends, so the ends of the distal portions were exposed to trypsin for 24 hours. The tapered and knotted ends were not evident on the trypsin-exposed preparations, suggesting that the ruptured ends contained a substantial amount of denatured collagen.

Accepted for publication 23 March, 1979

*Presented at the Biological Engineering Society Annual Day Conference 'Tendons and ligaments-natural and artificial', Leeds, 6 January, 1978.

Correspondence to Dr R. J. Minns, Department of Engineering Science, University of Durham, South Road, Durham DH1 3LE.
We ruptured human extensor tendons and, together with observing the ends in the scanning electron microscope, conducted fluorimetry assays of the unbroken and broken collagen, this being related to the denaturation that occurred within the tendon.

\section{Material and methods}

Extensor tendons of the hand were obtained from 3 males (aged 23, 29, and 41 years) at necropsy who had no known history of connective tissue disorders before death, and stored at $4^{\circ} \mathrm{C}$ until ready for testing. Lengths of tendon of approximately $6 \mathrm{~cm}$ were pulled in tension while wet until failure at an unknown strain rate in clamps at room temperature $\left(21^{\circ} \mathrm{C}\right)$. A portion near the cut end and the ruptured ends were immersed in a solution containing a proteolytic enzyme (trypsin, TPCK, Worthington Chemicals Inc., New Jersey, USA). The portion near the cut end acted as a control segment for the ruptured end (Fig. 1). After 15 minutes at $37^{\circ} \mathrm{C}$ the fluid in both tubes which contained the 2 sections of tendon were removed by pipette for assay. The same volume of fluid removed $(7 \mathrm{ml})$ was replaced in the numbered tubes, but this time containing $1 \%$ solution of eosin for staining the tendon. This showed on the removed tendon the amount of tendon that was immersed and exposed to the trypsin solution. The stained sections were cut, removed, dried, and weighed.

$1 \mathrm{ml}$ of each of the trypsin digests was mixed with $0.5 \mathrm{ml}$ acetone containing $150 \mu \mathrm{g}$ fluram and mixed 


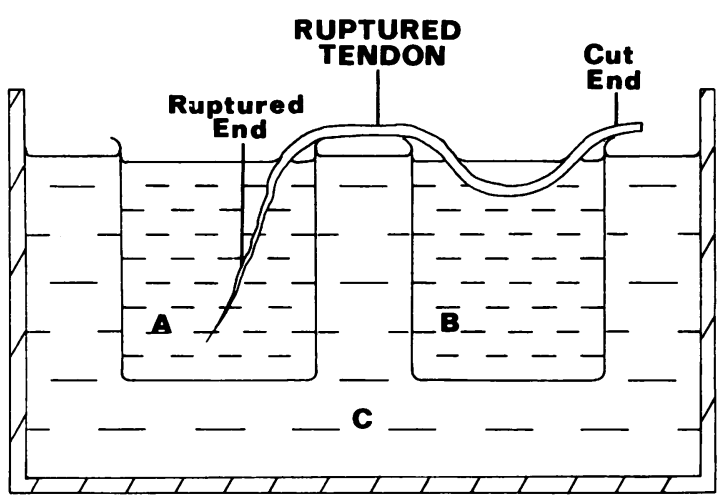

Fig. 1 Water bath containing water heated to $37^{\circ} \mathrm{C}$ ('C') and 2 series of tubes. The tubes in 'A' group included the ruptured tendon ends, while group 'B' had a middle portion of the same tendon. The fluids in ' $\mathrm{A}$ ' and ' $\mathrm{B}$ ' first contained $7 \mathrm{ml}$ of $1 \%$ sodium bicarbonate which included $2 \mathrm{mg}$ of trypsin. After 15 minutes the tubes were emptied, the supernatant being used in the fluram analysis, and then filled with $7 \mathrm{ml}$ of $1 \%$ eosin in water. The stained tendons were then removed and cut

to develop fluorescent derivatives of the free amino groups of the derived peptides. The assay was conducted on an Aminco bowman fluorimeter, giving the assay of peptide bonds per weight of tissue cleaved by trypsin, which should be greater in the denatured form.

The surface of the articular cartilage from osteoarthritic femoral heads was observed in the scanning electron microscope as described by the preparation of Minns et al. (1977).

Knee menisci were obtained at meniscectomy from 70 males (average age $28 \mathrm{yr}$ ) and cut into segments approximately $1 \mathrm{~cm}^{2}$, which included areas that had obvious degeneration as the result of trauma. The surface features of the menisci were observed in the scanning electron microscope.

Specimens for examination in the scanning electron microscope were dried by means of successively increasing strengths of acetone and finally dried with a critical point drying apparatus with carbon dioxide as the drying liquid. The specimens were mounted on marked aluminium stubs, coated with gold/palladium, and examined in a Cambridge S600 stereoscan at an accelerating voltage of $15 \mathrm{KV}$.

\section{Results}

The results of the fluram analysis indeed show that there is a significant increase in the denaturation that has occurred in the ruptured region compared to a section well away from the end and not including the end, the ratio of the fluram values being 3 times larger at the rupture site than in the control segment (Table 1).

The histology does not show this local phenomenon even for the tendon end that has been exposed to trypsin for a long time. However, the ruptured ends observed in the scanning electron microscope indeed show the end to result in a knobble (Fig. 2), which may well be the result of a recoil effect, but has certainly been the result of a tensile effect and contains a large amount of denatured collagen.

When collagen fibres were examined in pathological material, in particular arthritic articular cartilage and menisci, the fibrous surface contained many knotted fibre ends which may have undergone tensile failure. The surface of articular cartilage of osteoarthritic femoral heads removed at surgery for total hip replacement shows many bundles of

Table 1 Results of the fluram analysis on the ruptured ends and middle sections of failed tendons. The control was the supernatant that did not contain any tendon material

\begin{tabular}{llccc}
\hline & $\begin{array}{l}\text { Dry } \\
\text { weight of } \\
\text { tissue }(\mathrm{mg})\end{array}$ & $\begin{array}{l}\text { Fluram } \\
\text { in } 1 \mathrm{ml}\end{array}$ & $\begin{array}{l}\text { Fluram } \\
\text { per } 7 \mathrm{ml} \\
(\mathrm{F})\end{array}$ & $\begin{array}{l}\text { Fluram per } \\
\text { dry weight } \\
\text { of tissue }\end{array}$ \\
\hline Control & & 140 & 0 & 0 \\
Ruptured tendon ends & $1 \cdot 5$ & 390 & 1750 & 1166 \\
& 0.8 & 258 & 826 & 1033 \\
& $1 \cdot 3$ & 290 & 1050 & 807 \\
& $6 \cdot 8$ & 580 & 3080 & 496 \\
Mean values & $1 \cdot 2$ & 230 & 630 & 525 \\
& $2 \cdot 3$ & 350 & 1467 & 805 \\
Middle portion of & $2 \cdot 7$ & 245 & 735 & 272 \\
tendons & $2 \cdot 6$ & 265 & 875 & 336 \\
& $3 \cdot 7$ & 250 & 770 & 208 \\
& 1.9 & 200 & 420 & 221 \\
Mean values & 1.7 & 210 & 420 & 288 \\
& 2.5 & 234 & 658 & 265 \\
\hline
\end{tabular}

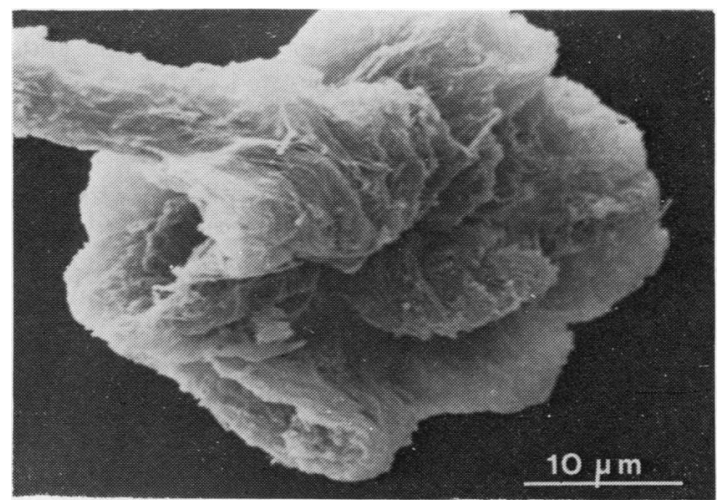

Fig. 2 Scanning electron micrograph of the knobble end of a mechanically ruptured extensor tendon 


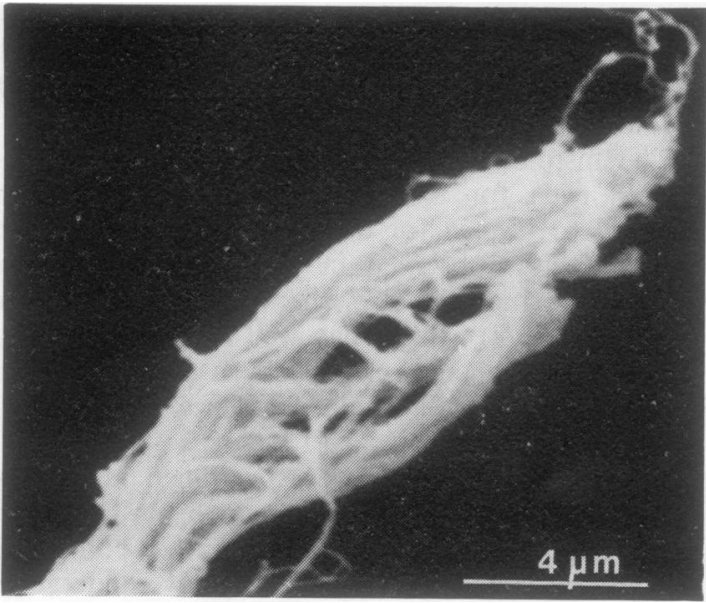

Fig. 3 Scanning electron micrograph of the torn end of a fibre bundle seen in osteoarthritic articular cartilage

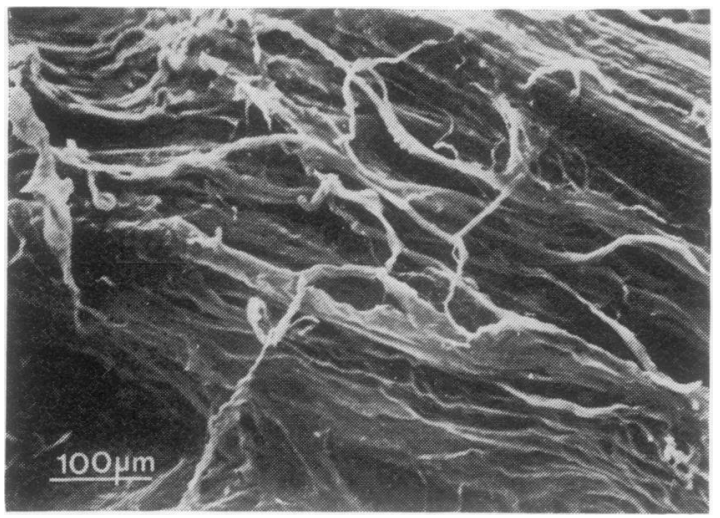

Fig. 4 Scanning electron micrograph of the surface of a torn knee meniscus showing torn and frayed collagen bundles

torn collagen fibres (Minns, et al., 1977), the ends of which contain fibrils with blunted ends, which no doubt contain a large volume of denatured material (Fig. 3). Similar areas can be seen as the surface of knee menisci with large bundles of torn collagen fibres (Fig. 4). An extensive amount of tensile rupturing has no doubt taken place.

\section{Discussion}

The biochemical processes involved in the repair of a ruptured tendon are complex, and we can only guess at some of these processes from evidence produced by in vitro studies. Two events must be fundamental in this repair process: $(a)$ the invasion of the site by phagocytic cells followed by fibroblasts, (b) the removal of the knotted ends of the ruptured collagen fibrils seen in the scanning electron microscope studies.

The removal of the denatured collagen molecules from the knotted fibril ends can readily be carried $\frac{\bar{\omega}}{\overrightarrow{0}}$ out in-vitro by the addition of a proteolytic enzyme, $\stackrel{\mathbb{Q}}{\Omega}$ for example, trypsin, In vivo the position is much ڤ more complicated, since serum inhibition of neutral $\overrightarrow{ }$ proteases occurs. Thus the removal of the denatured collagen is dependent on the balance of natural $\vec{\omega}$ protease activity on the one hand and inhibition $\stackrel{\circ}{\circ}$ of this activity on the other. The complex kinetics $\frac{\mu}{2}$ of a similar system have been fully analysed (Steven et al., 1978a) and verified by computer analysis $\stackrel{0}{\mathrm{i}}$ (Steven et al., 1978b).

The replacement of the broken ends of the tendon $\stackrel{\vec{A}}{\oplus}$ by repair collagen is typified by deposition of $ᄋ$ randomnly orientated collagen fibrils rather than $\rightarrow$ the aligned collagen fibrils observed in the intact $\frac{D}{2}$ portion of the tendon. During the process of tendon repair the extracellular medium also contains $\overrightarrow{0}$ mammalian collagenase and probably inhibitors $\infty^{\circ}$ of this enzyme (Sellers et al., 1977; Woolley et al., 1978). This collagenase attacks monomeric tropocollagen very much more rapidly than cross-linked molecules in fibrils and mature collagen fibres(Steven, 1976a). Thus the functions of the collagenase would seem to be the selective disruption of tropocollagen molecules which have not already formed fibrils. The end result is preferential retention of stabilised collagen molecules in fibril formation, which will ultimately mature and fill the gap between the ruptured fibre ends.

In such a scheme the timing of the collagenase attack is crucial. If this takes place too early, the repair process will be hindered, and if the attack takes place too late the region of the broken ends will be filled with randomnly orientated fibres and tendinous adhesions to the surrounding connective tissue. The role of collagenase inhibitors is also of importance, since the collagenolytic activity is a balance between enzyme activity opposed by inhibitor activity.

It is possible that an advantage could be obtained by deliberately preventing the overgrowth of collagen during repair to ruptured tendons, if this resulted in the organised regeneration of collagen fibrils from the proximal ends in a linear fashion parallel to the tendon's long axis. It is quite possible that in-vivo inhibitors of neutral proteases prevent the effective action of these enzymes, so that little of the denatured collagen is removed. In such a situation the alignment of newly synthesised tropocollagen molecules on existing collagen molecules of the tendon stump could not take place, owing to the presence of denatured collagen molecules. 
It may be possible to overcome this situation by injecting a solution of trypsin directly into the region of the stump ends, so as to overcome the local excess of inhibitor by increasing the concentration of neutral protease with additional trypsin, which also binds the inhibitor (Steven et al., 1978a). If this treatment proves to be capable of cleaning the stump ends of denatured collagen and exposing the native collagen fibril ends, there is a good chance that the repair process may be better orientated and result in a primary repair process taking place between the stump ends directed by the existing native fibrils protruding from the ends, initiating the laying down of parallel collagen fibrils.

\section{References}

Minns, R. J., Steven, F. S., and Hardinge, K. (1977). Osteoarthrotic articular cartilage lesions of the femoral head observed in the scanning electron microscope. Journal of Pathology, 122, 63-70.

Peacock, E. E. (1977). Some biologic and technical considerations in the repair of long tendons. Orthopaedic Clinics of North America, 8, 449-472.
Sellers, A., Cartwright, E., Murphy, G., and Reynolds, J. J. (1977). Evidence that latent collagenases are enzymeinhibitor complexes. Biochemical Journal, 163, 303-307.

Steven, F. S., Minns, R. J., and Finlay, J. B. (1975). Evidence for the local denaturation of collagen fibrils during the mechanical rupture of human tendons. Injury, 6, 317-320.

Steven, F. S. (1976a). Observations on the different substrate behaviour of tropocollagen molecules in solution and intermolecularly cross-linked tropocollagen within insoluble polymeric collagen fibrils. Biochemical Journal, 155, 391-400.

Steven, F. S. (1976b). Polymeric collagen fibrils. An example of substrate-mediated steric obstruction of enzyme degradation. Biochimica et Biophysica Acta, 452, 151-160.

Steven, F. S., Podrazky, V., and Itzhaki, S. (1978a). The interaction of a trypsin-dependent neutral protease and its inhibitor found in tumour cells. Analysis of complex kinetic data involved in a thioldisulphide exchange mechanism. Biochimica et Biophysica Acta, 524, 170-182.

Steven, F. S., Podrazky, V., and Foster, R. W. (1978b). Incremental analysis: the application to quantitation of both enzymic and inhibitory activity in complex subcellular fractions. Analytical Biochemistry, 90, 183-191.

Woolley, D. E., Akroyd, C., Evanson, J. M., Soames, J. V., and Davies, R. M. (1978). Characterisation and serum inhibition of neutral collagenase from cultured dog gingival tissue. Biochimica et Biophysica Acta, 522, 205217. 